\title{
Lie algebra and Laguerre Matrix Polynomials of One Variable
}

\author{
Ayman Shehata \\ Department of Mathematics, Faculty of Science, Assiut University, Assiut 71516, Egypt. \\ Department of Mathematics, College of Science and Arts in Unaizah, \\ Qassim University, Qassim, Kingdom of Saudi Arabia. \\ E-mail: drshehata20060yahoo.com
}

\begin{abstract}
The main object of this present paper is to introduce an extended family of Laguerre matrix polynomials and to derive a new class of certain summation formulas with the help of the Lie algebra method using techniques.
\end{abstract}

Keywords: Lie algebra, Laguerre matrix polynomials, Summation formulas.

2010 MSC No: 33C25; 15A60; 33C45.

\section{Introduction}

The analogous extension to the matrix framework for the classical case of Laguerre, modified Laguerre, and LaguerreKonhauser matrix polynomials have been studied in [1, 4, 6, 7, 9, 11, 12, 14, 15, 16, 17, 18. Indeed, in recent papers, Laguerre matrix polynomials have significant emergent. Some results and properties of classical Laguerre matrix functions have been extended to Laguerre matrix polynomials given in [2, 3, 8, 10, Our main aim in this study is to construct an extended family of Laguerre matrix polynomials and to discuss a new class of certain summation formulas of these matrix functions with the help of the Lie algebra method.

To achieve the purpose of this work, we recall here definitions and properties, which will be used below. Complex space $\mathbb{C}^{N \times N}$ of all square complex matrices of common order $N$ are considered.

Definition 1.1. [5] If $P$ is a positive stable matrix in $\mathbb{C}^{N \times N}$, then the Gamma matrix function $\Gamma(P)$ is defined by

$$
\Gamma(P)=\int_{0}^{\infty} e^{-t} t^{P-I} d t ; \quad t^{P-I}=\exp ((P-I) \ln t)
$$

where $I$ is an identity matrix in $\mathbb{C}^{N \times N}$.

From [5] if $A+n I$ is an invertible matrix for all integers $n \geq 0$ then the matrix version of the Pochhammer symbol is

$$
(A)_{n}=A(A+I) \ldots(A+(n-1) I)=\Gamma(A+n I) \Gamma^{-1}(A) ; n \geq 1 ;(A)_{0}=I,
$$

and $\Gamma(A)$ is an invertible matrix, its inverse coincides with $\Gamma^{-1}(A)$.

Definition 1.2. (Jódar and Sastre [3]) Let $A$ be a matrix in $\mathbb{C}^{N \times N}$ satisfying the condition

$$
-k \notin \sigma(A) \text { for every integer } k>0 \text {, }
$$

where $\sigma(A)$ is the set of all eigenvalues of $A$ and $\lambda$ is a complex number whose real part is a positive number. Then the Laguerre matrix polynomials is defined by

$$
L_{n}^{(A, \lambda)}(x)=\sum_{k=0}^{n} \frac{(-1)^{k}(A+I)_{n}\left[(A+I)_{k}\right]^{-1}(\lambda x)^{k}}{k !(n-k) !} ; \lambda \geq 0 .
$$


We recall that the properties of Laguerre matrix polynomials are

$$
[n I-x D I] L_{n}^{(A, \lambda)}(x)=(A+n I) L_{n-1}^{(A, \lambda)}(x) ; n \geq 1
$$

and

$$
[x D I+(A+(n+1-\lambda x) I)] L_{n}^{(A, \lambda)}(x)=(n+1) L_{n+1}^{(A, \lambda)}(x) .
$$

\section{Group-Theoretic discussion for Laguerre matrix polynomials}

By starting with the equations (5) and (6) give the raising and lowering operators $\mathbb{A}$ and $\mathbb{B}$ with respect to the index $n$. We consider a spurious variable $y$ and define Laguerre matrix polynomials $\Phi_{n}^{(A, \lambda)}(x, y)$ in the form

$$
\Phi_{n}^{(A, \lambda)}(x, y)=e^{n y} L_{n}^{(A, \lambda)}(x), n \in \mathbb{N}
$$

In the present investigation for local transformation of Lie groups, we have the following matrix recurrence relations:

$$
\begin{gathered}
\mathbb{A} \Phi_{n}^{(A, \lambda)}(x, y)=(A+n I) \Phi_{n-1}^{(A, \lambda)}(x, y), \\
\mathbb{B} \Phi_{n}^{(A, \lambda)}(x, y)=(n+1) \Phi_{n+1}^{(A, \lambda)}(x, y),
\end{gathered}
$$

and

$$
\mathbb{C} \Phi_{n}^{(A, \lambda)}(x, y)=(A+(2 n+1) I) \Phi_{n}^{(A, \lambda)}(x, y)
$$

where the first order linear differential operators

$$
\begin{gathered}
\mathbb{A}=\left[\frac{\partial}{\partial y}-x \frac{\partial}{\partial x}\right] e^{-y} I, \\
\mathbb{B}=\left[A+\left(x \frac{\partial}{\partial x}+1-\lambda x+\frac{\partial}{\partial y}\right) I\right] e^{y},
\end{gathered}
$$

and

$$
\mathbb{C}=2 \frac{\partial}{\partial y} I+A+I
$$

From the above commutator relations, we can state the next theorem.

Theorem 2.1. Differential operators $\mathbb{A}, \mathbb{B}$ and $\mathbb{C}$ satisfy the commutation relations

$$
(i)[\mathbb{A}, \mathbb{B}]=\mathbb{C}, \quad(\text { ii })[\mathbb{A}, \mathbb{C}]=2 \mathbb{A}, \quad(\text { iii })[\mathbb{B}, \mathbb{C}]=-2 \mathbb{B} .
$$

Proof. Now, we find that

$$
\mathbb{A} \mathbb{B} \Phi_{n}^{(A, \lambda)}(x, y)=\left[\frac{\partial}{\partial y}-x \frac{\partial}{\partial x}\right] e^{-y}\left[\left[A+\left(x \frac{\partial}{\partial x}+1-\lambda x+\frac{\partial}{\partial y}\right) I\right] e^{y}\right] \Phi_{n}^{(A, \lambda)}(x, y) .
$$

Hence, on simplification, we get

$$
\begin{aligned}
\mathbb{A B} \Phi_{n}^{(A, \lambda)}(x, y)= & \lambda x \Phi_{n}^{(A, \lambda)}(x, y)+(A+(2-\lambda x) I) \frac{\partial}{\partial y} \Phi_{n}^{(A, \lambda)}(x, y)+\frac{\partial^{2}}{\partial y^{2}} \Phi_{n}^{(A, \lambda)}(x, y) \\
& -(A+(3-\lambda x) I) \frac{\partial}{\partial x} x \Phi_{n}^{(A, \lambda)}(x, y)-x^{2} \frac{\partial^{2}}{\partial x^{2}} \Phi_{n}^{(A, \lambda)}(x, y) .
\end{aligned}
$$

On the other hand, we get

$$
\mathbb{B} \mathbb{A} \Phi_{n}^{(A, \lambda)}(x, y)=\left[A+\left(x \frac{\partial}{\partial x}+1-\lambda x+\frac{\partial}{\partial y}\right) I\right] e^{y}\left[\frac{\partial}{\partial y}-x \frac{\partial}{\partial x}\right] e^{-y} \Phi_{n}^{(A, \lambda)}(x, y) .
$$


This is to be simplified as

$$
\begin{aligned}
& \mathbb{B} \mathbb{A} \Phi_{n}^{(A, \lambda)}(x, y)=-(A+(1-\lambda x) I) \Phi_{n}^{(A, \lambda)}(x, y)+(A-\lambda x I) \frac{\partial}{\partial y} \Phi_{n}^{(A, \lambda)}(x, y) \\
& +\frac{\partial^{2}}{\partial y^{2}} \Phi_{n}^{(A, \lambda)}(x, y)-(A+(3-\lambda x) I) \frac{\partial}{\partial x} x \Phi_{n}^{(A, \lambda)}(x, y)-x^{2} \frac{\partial^{2}}{\partial x^{2}} \Phi_{n}^{(A, \lambda)}(x, y) .
\end{aligned}
$$

Then for $\frac{\partial^{2}}{\partial x \partial y}=\frac{\partial^{2}}{\partial y \partial x}$, if we subtract 15 from 16 , we have

$$
[\mathbb{A}, \mathbb{B}] \Phi_{n}^{(A, \lambda)}(x, y)=(A+I) \Phi_{n}^{(A, \lambda)}(x, y)+2 \frac{\partial}{\partial y} \Phi_{n}^{(A, \lambda)}(x, y) .
$$

Thus, we have the required result $[\mathbb{A}, \mathbb{B}]=\mathbb{C}$.

Secondly in order to prove (ii). In a similar manner, we find

$$
\mathbb{A} \mathbb{C} \Phi_{n}^{(A, \lambda)}(x, y)=\left[\frac{\partial}{\partial y}-x \frac{\partial}{\partial x}\right] e^{-y}\left[A+I+2 \frac{\partial}{\partial y} I\right] \Phi_{n}^{(A, \lambda)}(x, y) .
$$

which can be simplified as

$$
\begin{aligned}
& \mathbb{A} \mathbb{C} \Phi_{n}^{(A, \lambda)}(x, y)=(A+I) \frac{\partial}{\partial y}\left(e^{-y} \Phi_{n}^{(A, \lambda)}(x, y)\right)-2 e^{-y} \frac{\partial}{\partial y} \Phi_{n}^{(A, \lambda)}(x, y) \\
& +2 e^{-y} \frac{\partial^{2}}{\partial y^{2}} \Phi_{n}^{(A, \lambda)}(x, y)-(A+I) x e^{-y} \frac{\partial}{\partial x} \Phi_{n}^{(A, \lambda)}(x, y)-2 x e^{-y} \frac{\partial^{2}}{\partial x \partial y} \Phi_{n}^{(A, \lambda)}(x, y),
\end{aligned}
$$

and

$$
\begin{aligned}
& \mathbb{C} \mathbb{A} \Phi_{n}^{(A, \lambda)}(x, y)=\left[A+I+2 \frac{\partial}{\partial y} I\right]\left[\frac{\partial}{\partial y}-x \frac{\partial}{\partial x}\right] e^{-y} \Phi_{n}^{(A, \lambda)}(x, y) \\
& =(A-I) \frac{\partial}{\partial y}\left(e^{-y} \Phi_{n}^{(A, \lambda)}(x, y)\right)-2 e^{-y} \frac{\partial}{\partial y} \Phi_{n}^{(A, \lambda)}(x, y) \\
& +2 e^{-y} \frac{\partial^{2}}{\partial y^{2}} \Phi_{n}^{(A, \lambda)}(x, y)-(A-I) x e^{-y} \frac{\partial}{\partial x} \Phi_{n}^{(A, \lambda)}(x, y)-2 x e^{-y} \frac{\partial^{2}}{\partial x \partial y} \Phi_{n}^{(A, \lambda)}(x, y) .
\end{aligned}
$$

Combining (18) and 19, we get the result

$$
[\mathbb{A}, \mathbb{C}] \Phi_{n}^{(A, \lambda)}(x, y)=2 \frac{\partial}{\partial y}\left(e^{-y} \Phi_{n}^{(A, \lambda)}(x, y)\right)-2 x \frac{\partial}{\partial x}\left(e^{-y} \Phi_{n}^{(A, \lambda)}(x, y)\right) .
$$

Hence, we have

$$
[\mathbb{A}, \mathbb{C}]=2 \mathbb{A}
$$

Lastly, we consider

$$
\mathbb{B} C \Phi_{n}^{(A, \lambda)}(x, y)=\left[A+\left(x \frac{\partial}{\partial x}+1-\lambda x+\frac{\partial}{\partial y}\right) I\right] e^{y}\left[A+I+2 \frac{\partial}{\partial y} I\right] \Phi_{n}^{(A, \lambda)}(x, y),
$$

which can be simplified as

$$
\begin{aligned}
& \mathbb{B} \mathbb{C} \Phi_{n}^{(A, \lambda)}(x, y)=(A+I)(A+(1-\lambda x) I) e^{y} \Phi_{n}^{(A, \lambda)}(x, y) \\
& +(A+I) \frac{\partial}{\partial y}\left(e^{y} \Phi_{n}^{(A, \lambda)}(x, y)\right)+2(A+(2-\lambda x) I) e^{y} \frac{\partial}{\partial y} \Phi_{n}^{(A, \lambda)}(x, y) \\
& +2 e^{y} \frac{\partial^{2}}{\partial y^{2}} \Phi_{n}^{(A, \lambda)}(x, y)+(A+I) x e^{y} \frac{\partial}{\partial x} \Phi_{n}^{(A, \lambda)}(x, y)+2 x e^{y} \frac{\partial^{2}}{\partial x \partial y} \Phi_{n}^{(A, \lambda)}(x, y) .
\end{aligned}
$$

Similarly, we have

$$
\begin{aligned}
& \mathbb{C B} \Phi_{n}^{(A, \lambda)}(x, y)=(A+I)(A+(2-\lambda x) I) e^{y} \Phi_{n}^{(A, \lambda)}(x, y) \\
& +2(A+(2-\lambda x) I) \frac{\partial}{\partial y}\left(e^{y} \Phi_{n}^{(A, \lambda)}(x, y)\right)+(A+3 I) e^{y} \frac{\partial}{\partial y} \Phi_{n}^{(A, \lambda)}(x, y) \\
& +2 e^{y} \frac{\partial^{2}}{\partial y^{2}} \Phi_{n}^{(A, \lambda)}(x, y)+(A+3 I) x e^{y} \frac{\partial}{\partial x} \Phi_{n}^{(A, \lambda)}(x, y)+2 x e^{y} \frac{\partial^{2}}{\partial x \partial y} \Phi_{n}^{(A, \lambda)}(x, y) .
\end{aligned}
$$


Subtracting 212 from 22), we get

$$
[\mathbb{B}, \mathbb{C}] \Phi_{n}^{(A, \lambda)}(x, y)=-2\left[A+\left(x \frac{\partial}{\partial x}+1-\lambda x+\frac{\partial}{\partial y}\right) I\right]\left(e^{y} \Phi_{n}^{(A, \lambda)}(x, y)\right) .
$$

Hence, we have the commutation relation

$$
[\mathbb{B}, \mathbb{C}]=-2 \mathbb{B},
$$

which completes the proof of the theorem.

\section{Applications}

Here the summation formulas of Laguerre matrix polynomials 77 have been derived from the finite differential operator $e^{-\alpha A}$. It is interesting to notice that while constructing the Lie-algebra for Laguerre matrix polynomials, we have

$$
e^{-\alpha \mathbb{A}} x=\frac{x}{1-\alpha e^{-y}},\left|\alpha e^{-y}\right|<1
$$

and

$$
e^{-\alpha \mathbb{A}} y=y+\log \left(1-\alpha e^{-y}\right)
$$

Thus

$$
\begin{aligned}
e^{-\alpha \mathbb{A}} \Phi_{n}^{(A, \lambda)}(x, y) & =\Phi_{n}^{(A, \lambda)}\left(\frac{x}{1-\alpha e^{-y}}, y+\log \left(1-\alpha e^{-y}\right)\right) \\
& =e^{n y}\left(1-\alpha e^{-y}\right)^{n} L_{n}^{(A, \lambda)}\left(\frac{x}{1-\alpha e^{-y}}\right) .
\end{aligned}
$$

On the other hand, from eq. (8), we have

$$
\begin{aligned}
& e^{-\alpha \mathbb{A}} \Phi_{n}^{(A, \lambda)}(x, y)=e^{n y} \sum_{k=0}^{n} \frac{(-\alpha)^{k}}{k !} e^{-k y} \\
& \times(A+n I)(A+(n-1) I) \ldots(A+(n-k+1) I) L_{n-k}^{(A, \lambda)}(x) .
\end{aligned}
$$

Writing $t=-\alpha e^{-y}$ and $|t|<1$, we obtain a well-known finite sum for the Laguerre matrix polynomials

$$
\begin{aligned}
& (1+t)^{n} L_{n}^{(A, \lambda)}\left(\frac{x}{1+t}\right) \\
& =\sum_{k=0}^{n} \frac{t^{k}}{k !}(A+n I)(A+(n-1) I) \ldots(A+(n-k+1) I) L_{n-k}^{(A, \lambda)}(x) .
\end{aligned}
$$

\section{Conclusion}

These are the main results of investigating the family of Laguerre matrix polynomials using Lie algebra method. Starting from the modified forms of the matrix differential recurrence relations of Laguerre matrix polynomials are one of these direct methods and clearly some directions to develop more researchers and studies in that area. Also, some interested summation formulas and consequences of our results have been discussed. The results of this paper are original, variant, significant and so it is interesting and capable to develop its study in the future which plays the vital role in Mathematical Physics.

Acknowledgments. (a) The Author wishes to express his sincere appreciation to Dr. Shimaa Ibrahim Moustafa Abdal-Rahman, (Department of Mathematics, Faculty of Science, Assiut University, Assiut 71516, Egypt) for his kind interest, help, encouragements, and the investigations for this paper.

(b) The author would like to thank the anonymous referees for their helpful comments and suggestions which improved the original version of this paper. 


\section{References}

[1] R. Agarwal, and S. Jain, Certain properties of some special matrix functions via Lie Algebra. International Bulletin of Mathematical Research, Vol. 2, No. 1 (2015), pp.9-15.

[2] L. Jódar, R. Company, E. and Navarro, Laguerre matrix polynomials and system of second order differential equations. Applied Numerical Mathematics, Vol. 15, No. 1 (1994), pp.53-63.

[3] L. Jódar, and J. Sastre, On Laguerre matrix polynomials. Utilitas Mathematica, Vol. 53 (1998), pp.37-48.

[4] S. Khan, and N.A.M. Hassan, 2-Variable Laguerre matrix polynomials and Lie-algebraic techniques. Journal of Physics A: Mathematical and Theoretical, Vol. 43, No. 23 (2010), 235204 (21pp).

[5] L. Jódar, and J.C. Cortés, Some properties of Gamma and Beta matrix functions. Applied Mathematics Letters, Vol. 11, No. 1 (1998), pp.89-93.

[6] M.J.S. Shahwan, and M.A. Pathan, Origin of certain generating relations of Hermite matrix functions from the view point of Lie Algebra. Integral Transform and Special Functions, Vol. 17, No. 10 (2006), pp.743-747.

[7] M.J.S. Shahwan, and M.A. Pathan, Generating relations of Hermite matrix polynomials by Lie Algebraic method. Italian Journal of pure and Applied Mathematics. Vol. 25 (2009), pp.187-192.

[8] J. Sastre, and L. Jódar, On Laguerre matrix polynomials series. Utilitas Mathematica, Vol. 71 (2006), pp.109130.

[9] A. Shehata, Connections between Legendre with Hermite and Laguerre matrix polynomials. Gazi University Journal of Science, Vol. 28, No. 2 (2015), pp.221-230.

[10] A. Shehata, Some relations on Laguerre matrix polynomials. Malaysian Journal of Mathematical Sciences, Vol. 9, No. 3 (2015), pp.443-462.

[11] A.Shehata, On modified Laguerre matrix polynomials. Journal of Natural Sciences and Mathematics, Vol. 8, No. 2 (2015), pp.153-166.

[12] A. Shehata, Certain generating relations of generalized Bessel matrix polynomials from the view point of Lie algebra method. Bulletin of the Iranian Mathematical Society, (in press).

[13] A. Shehata, Certain generating relations of Konhauser matrix polynomials from the view point of Lie algebra method. University Politechnica of Bucharest Scientific Bulletin- series A-Applied mathematics and physics, Vol. 79, No. 4 (2017), pp.123-136.

[14] B. Çekim, and A. Altin, New matrix formulas for Laguerre matrix polynomials, Journal of Classical Analysis, Vol. 3, No. 1 (2013), pp.59-67.

[15] M.G. Bin-Saad and A.A. Al-Sayaad, Study of two variable Laguerre matrix polynomials via symbolic operational images. Asian Journal of Mathematics and Computer Research, Vol. 2, No. 1 (2015), pp.42-50.

[16] M.G. Bin-Saad, A note on two variable Laguerre matrix polynomials. Journal of the Association of Arab Universities for Basic and Applied Sciences, Vol. 24 (2017), pp.271-276.

[17] A.Cevik, and A. Alitn, A note on Laguerre matrix polynomials. Mathematical Sciences And Applications ENotes, Vol. 3, No. 2 (2015), pp.54-57.

[18] Z. Zhu and Z. Li, A note on sobolev orthogonality for Laguerre matrix polynomials. Analysis in Theory and Applications Vol. 23, No. 1 (2007), pp.26-34. 